\title{
CONSISTENCY OF SAMPLE ESTIMATES OF RISK AVERSE STOCHASTIC PROGRAMS
}

\author{
ALEXANDER SHAPIRO, ${ }^{*}$ Georgia Institute of Technology
}

\begin{abstract}
In this paper we study asymptotic consistency of law invariant convex risk measures and the corresponding risk averse stochastic programming problems for independent, identically distributed data. Under mild regularity conditions, we prove a law of large numbers and epiconvergence of the corresponding statistical estimators. This can be applied in a straightforward way to establish convergence with probability 1 of samplebased estimators of risk averse stochastic programming problems.
\end{abstract}

Keywords: Law invariant convex and coherent risk measures; stochastic programming; law of large numbers; consistency of statistical estimators; epiconvergence; sample average approximation

2010 Mathematics Subject Classification: Primary 62F12

Secondary $90 \mathrm{C} 15$

\section{Introduction}

In many practical situations one has to make decisions about uncertainty in the future. This raises the question of how to make such decisions in some optimal way, i.e. how to formulate an appropriate optimization problem. In the classical approach of stochastic programming one specifies a probability distribution of the uncertain parameters and optimizes (say minimizes) a relevant objective function on average. Since this does not take into account deviations of the possible realization of the objective from its expected value, this approach is referred to as risk neutral. Recently, considerable attention has been paid to risk averse formulations of stochastic programs.

To be specific, let us consider the optimization problem

$$
\min _{x \in \mathcal{X}} G(x, \xi)
$$

depending on parameter vector $\xi \in \Xi \subset \mathbb{R}^{d}$. Here $\chi \subset \mathbb{R}^{n}$ is the feasible set of decision variables and $G: \mathcal{X} \times \mathbb{R}^{d} \rightarrow \mathbb{R}$ is the objective function. An optimal solution of this problem depends on a particular realization of $\xi$, which is not known at the time when decision $x$ should be made. If $\xi$ is modeled as a random vector with a specified probability distribution then we can average the objective by taking the expectation $g(x):=\mathbb{E}[G(x, \xi)]$, and, consequently, minimize $g(x)$ subject to the feasibility constraints $x \in \mathcal{X}$. If this procedure is repeated many times, under more or less similar conditions, such a decision will be optimal on average and can be justified by the law of large numbers. However, for a particular realization of the random vector $\xi$ and given $x$, the value of random variable $G_{x}(\xi)=G(x, \xi)$ can be quite different from the expected value $g(x)$. This gives a motivation for considering an appropriate functional $\rho$

Received 27 April 2012; revision received 29 September 2012.

* Postal address: Georgia Institute of Technology, School of Industrial \& Systems Engineering, 765 Ferst Drive, Atlanta, GA 30332, USA. Email address: ashapiro@isye.gatech.edu 
other than the expectation, defined on a space of random variables, and, hence, considering the corresponding risk averse problem (see, e.g. [8, Chapter 6])

$$
\min _{x \in \mathcal{X}}\left\{g(x):=\rho\left(G_{x}\right)\right\} .
$$

In order to formalize the concept of risk functionals, we proceed as follows. Suppose that the random vector $\xi=\xi(\omega)$ is defined on a probability space $(\Omega, \mathcal{F}, \mathbb{P})$. Let $\mathcal{Z}:=$ $L_{p}(\Omega, \mathcal{F}, \mathbb{P}), p \in[1, \infty)$, be the space of random variables $Z: \Omega \rightarrow \mathbb{R}$ having finite $p$ th-order moments, and let $\rho: \mathcal{Z} \rightarrow \mathbb{R}$ be a (real-valued) functional referred to as a risk measure. Assume that the random variable $G_{x}(\xi(\omega))$ belongs to the space $\mathcal{Z}$ for all $x \in \mathcal{X}$; hence, $\rho\left(G_{x}\right)$ is well defined.

In applications the functional $\rho$ is often constructed in such a way as to provide a compromise between optimization on average and controlling the involved risk. Therefore, something like 'mean risk' could be a better term for $\rho$. However, the terminology of risk measures has become somewhat standard, so we will follow it here. In the influential paper of Artzner et al. [2] it was suggested that a 'good' risk measure should satisfy the following conditions (axioms).

(A1) Monotonicity. If $Z, Z^{\prime} \in \mathcal{Z}$ and $Z \succeq Z^{\prime}$, then $\rho(Z) \geq \rho\left(Z^{\prime}\right)$.

(A2) Convexity.

$$
\rho\left(t Z+(1-t) Z^{\prime}\right) \leq t \rho(Z)+(1-t) \rho\left(Z^{\prime}\right)
$$

for all $Z, Z^{\prime} \in \mathcal{Z}$ and all $t \in[0,1]$.

(A3) Translation equivariance. If $a \in \mathbb{R}$ and $Z \in \mathcal{Z}$, then $\rho(Z+a)=\rho(Z)+a$.

(A4) Positive homogeneity. If $t \geq 0$ and $Z \in \mathcal{Z}$, then $\rho(t Z)=t \rho(Z)$.

The notation $Z \succeq Z^{\prime}$ means that $Z(\omega) \geq Z^{\prime}(\omega)$ for almost every (a.e.) $\omega \in \Omega$. Risk measures $\rho$ satisfying axioms (A1)-(A4) were called coherent in [2]. If a risk measure satisfies axioms (A1)-(A3), but not necessarily (A4), it is called convex (cf. [5]). It is said that $\rho$ is law invariant if $\rho(Z)$ depends only on the distribution of $Z$, i.e. if $Z, Z^{\prime} \in \mathcal{Z}$ have the same cumulative distribution function then $\rho(Z)=\rho\left(Z^{\prime}\right)$. Throughout the paper, we denote by $F(z):=\mathbb{P}(Z \leq z)$ the cumulative distribution function (CDF) of a considered random variable $Z \in \mathcal{Z}$.

An important example of risk measures is the average value-at-risk (also called the conditional value-at-risk) measure

$$
\operatorname{AVaR}_{\alpha}(Z):=\inf _{t \in \mathbb{R}}\left\{t+(1-\alpha)^{-1} \mathbb{E}[Z-t]_{+}\right\}
$$

where $\alpha \in[0,1)$. Defined on $\mathcal{Z}:=L_{1}(\Omega, \mathcal{F}, \mathbb{P})$, this is a (real-valued) law invariant coherent risk measure. Given a sample $Z_{1}, \ldots, Z_{N}$ of random variable $Z$, the corresponding empirical $\mathrm{CDF}$ is $\hat{F}_{N}(z)=N^{-1} \sum_{i=1}^{N} \mathbf{1}_{\left\{Z_{i} \leq z\right\}}$. Consequently, $\operatorname{AVaR}_{\alpha}(Z)$ can be estimated by replacing the CDF $F$ of $Z$ with its empirical estimate $\hat{F}_{N}$, that is, by replacing the expectation $\mathbb{E}[Z-t]_{+}$ in (1.2) with its sample average estimate $N^{-1} \sum_{i=1}^{N}\left[Z_{i}-t\right]_{+}$. We assume throughout this paper that the sample $Z_{1}, \ldots, Z_{N}$ is independent and identically distributed (i.i.d.). Since a law invariant risk measure $\rho$ can be considered as a function of the $\operatorname{CDF} F(z)=\mathbb{P}(Z \leq z)$, we also write $\rho(F)$ to denote the corresponding value $\rho(Z)$. By replacing $F$ with its empirical estimate $\hat{F}_{N}$, we obtain the estimate $\rho\left(\hat{F}_{N}\right)$ to which we refer as the sample or empirical estimate of $\rho(F)$.

In a similar way the 'true' risk averse optimization problem (1.1) can be approximated by its empirical estimate. That is, let $\xi_{i}=\xi_{i}(\omega), i=1, \ldots, N$, be an i.i.d. sample of the random 
vector $\xi=\xi(\omega)$, defined on the same probability space. For $x \in \mathbb{R}^{n}$, consider the sample estimate $\hat{g}_{N}(x)$ given by $\rho\left(\hat{F}_{N}\right)$, with $\hat{F}_{N}$ being the empirical CDF associated with the sample $G\left(x, \xi_{1}\right), \ldots, G\left(x, \xi_{N}\right)$. Consequently, we obtain the following approximation of problem (1.1):

$$
\operatorname{Min}_{x \in \mathcal{X}} \hat{g}_{N}(x)
$$

Note that $\hat{g}_{N}(x)=\hat{g}_{N}(x, \omega)$ is a random function-we sometimes suppress the dependence on $\omega$ in the notation.

The goal of this paper is to investigate convergence properties of the sample estimates of law invariant convex risk measures and the corresponding optimization problems of the form (1.3). A need for such statistical inference appears in two somewhat different situations. It could be that the random sample represents given (collected) data while the 'true' distribution is not known. On the other hand, in some cases the random sample is generated by Monte Carlo techniques for the purpose of numerical integration. In the context of solving the optimization problem (1.3) this is the approach of the so-called sample average approximation (SAA) method (see, e.g. [8, Chapter 5]). Although conceptually different these two applications involve the same statistical inference.

Let us recall the following basic duality result associated with convex risk measures. Recall that the space $\mathcal{Z}=L_{p}(\Omega, \mathcal{F}, \mathbb{P}), p \in[1, \infty)$, equipped with the norm $\|Z\|_{p}=$ $\left(\int_{\Omega}|Z(\omega)|^{p} \mathrm{~d} \mathbb{P}(\omega)\right)^{1 / p}$, becomes a Banach space, and its dual space is $\mathcal{Z}^{*}=L_{q}(\Omega, \mathcal{F}, \mathbb{P})$, where $q \in(1, \infty]$ is such that $1 / p+1 / q=1$. A (real-valued) convex risk measure $\rho: \mathcal{Z} \rightarrow \mathbb{R}$ is continuous (in the norm topology of $\mathcal{Z}$ ) and there exists a convex set $\mathfrak{A} \subset \mathfrak{Z}^{*}$ of probability density functions such that

$$
\rho(Z)=\sup _{\zeta \in \mathfrak{A}} \int_{\Omega} Z(\omega) \zeta(\omega) \mathrm{d} \mathbb{P}(\omega)-\rho^{*}(\zeta) \quad \text { for all } Z \in \mathcal{Z},
$$

where $\rho^{*}: \mathfrak{Z}^{*} \rightarrow \overline{\mathbb{R}}$ is the conjugate of the functional $\rho$ and $\mathfrak{A}=\operatorname{dom}\left(\rho^{*}\right)$. The dual representation (1.4) follows from the classical Fenchel-Moreau theorem. Originally, it was derived in [2], and the follow-up literature (cf. [5]), for the space $\mathcal{Z}=L_{\infty}(\Omega, \mathcal{F}, \mathbb{P})$. For spaces $\mathcal{Z}=L_{p}(\Omega, \mathcal{F}, \mathbb{P}), p \in[1, \infty)$, this representation was derived in [7] and it was shown there that monotonicity (axiom (A1)) and convexity (axiom (A2)) imply continuity of the (real-valued) risk measure $\rho$ in the norm topology of the space $L_{p}(\Omega, \mathcal{F}, \mathbb{P})$.

We assume throughout this paper that the probability space $(\Omega, \mathcal{F}, \mathbb{P})$ is atomless and complete. Then we can set $\Omega:=[0,1]$ equipped with its Lebesgue sigma-algebra $\mathcal{F}$ (cf. [3, p. 25]), and uniform probability distribution (measure) $\mathbb{P}$. For a $\operatorname{CDF} F$, we denote by $F^{-1}(\omega)=\inf \{z: F(z) \geq \omega\}$ its left-side quantile. Note that $Z(\cdot):=F^{-1}(\cdot)$ is a real-valued, monotonically nondecreasing, left-side continuous function on the interval $(0,1)$, and (in the case where $\Omega=[0,1])$ can be considered as an element of the space $L_{p}(\Omega, \mathcal{F}, \mathbb{P})$, provided that $\int_{0}^{1}|Z(\omega)|^{p} \mathrm{~d} \omega$ is finite.

\section{Law of large numbers for sample estimates of convex risk measures}

In this section we investigate convergence with probability 1 (w.p. 1) of sample estimates of convex risk measures.

Theorem 2.1. Let $\mathcal{Z}:=L_{p}(\Omega, \mathcal{F}, \mathbb{P}), p \in[1, \infty)$, and $\rho: \mathcal{Z} \rightarrow \mathbb{R}$ be a (real-valued) law invariant convex risk measure. Then $\rho\left(\hat{F}_{N}\right)$ converges to $\rho(F)$ w.p. 1 as $N \rightarrow \infty$. 
Proof. Recall that, since the space $(\Omega, \mathcal{F}, \mathbb{P})$ is assumed to be atomless and complete, we can work with $\Omega=[0,1]$ equipped with its Lebesgue sigma-algebra $\mathcal{F}$ and uniform probability distribution $\mathbb{P}$. Consider $Z(\cdot):=F^{-1}(\cdot)$. We can view $Z$ as an element of the space $\mathcal{Z}=L_{p}(\Omega, \mathcal{F}, \mathbb{P})$. Consider $\hat{Z}_{N}:=\hat{F}_{N}^{-1}$ to be the left-side quantile of the empirical $\mathrm{CDF} \hat{F}_{N}$ (based on i.i.d. sample $\left.Z_{1}, \ldots, Z_{N}\right)$. Recall that $\rho(Z)=\rho(F)$ and $\rho\left(\hat{Z}_{N}\right)=\rho\left(\hat{F}_{N}\right)$. Note that the function $\hat{Z}_{N}(\cdot)$ is piecewise constant and, hence, $\hat{Z}_{N} \in \mathcal{Z}$ as well. Consider the set $\mathfrak{C} \subset[0,1]$ of points where the function $Z(\cdot)$ is discontinuous. Since $Z(\cdot)$ is monotonically nondecreasing, the set $\mathfrak{C}$ is countable and, hence, has Lebesgue measure 0. By the GlivenkoCantelli theorem, w.p. $1, \hat{F}_{N}$ converges to $F$ uniformly on $\mathbb{R}$. It follows that, w.p. $1, \hat{Z}_{N}$ converges pointwise to $Z$ on the set $[0,1] \backslash \mathfrak{C}$, and, hence, w.p. 1 ,

$$
\lim _{N \rightarrow \infty} \int_{0}^{1}\left|Z(\omega)-\hat{Z}_{N}(\omega)\right|^{p} \mathrm{~d} \omega=\int_{0}^{1} \lim _{N \rightarrow \infty}\left|Z(\omega)-\hat{Z}_{N}(\omega)\right|^{p} \mathrm{~d} \omega=0,
$$

where the interchangeability of the limit and integral operators is justified provided that, w.p. 1, the sequence $\left|Z(\cdot)-\hat{Z}_{N}(\cdot)\right|^{p}$ is uniformly integrable (see, e.g. [3, p. 217]).

Let us show that the uniform integrability indeed holds. We have (triangle inequality) $\left\|Z-\hat{Z}_{N}\right\|_{p} \leq\|Z\|_{p}+\left\|\hat{Z}_{N}\right\|_{p}$, i.e.

$$
\left(\int_{0}^{1}\left|Z(\omega)-\hat{Z}_{N}(\omega)\right|^{p} \mathrm{~d} \omega\right)^{1 / p} \leq\left(\int_{0}^{1}|Z(\omega)|^{p} \mathrm{~d} \omega\right)^{1 / p}+\left(\int_{0}^{1}\left|\hat{Z}_{N}(\omega)\right|^{p} \mathrm{~d} \omega\right)^{1 / p}
$$

The first term on the right-hand side of (2.2) is constant; therefore, it is sufficient to verify uniform integrability of $\left|\hat{Z}_{N}(\cdot)\right|^{p}$. We have

$$
\int_{0}^{1}\left|\hat{Z}_{N}(\omega)\right|^{p} \mathrm{~d} \omega=\int_{0}^{\infty}|z|^{p} \mathrm{~d} \hat{F}_{N}(z)=\frac{1}{N} \sum_{i=1}^{N}\left|Z_{i}\right|^{p}
$$

and, by the law of large numbers, $N^{-1} \sum_{i=1}^{N}\left|Z_{i}\right|^{p}$ converges w.p. 1 to $\mathbb{E}|Z|^{p}$. Since $Z \in$ $L_{p}(\Omega, \mathcal{F}, \mathbb{P}), \mathbb{E}|Z|^{p}$ is finite. It follows that $\int_{0}^{1}\left|\hat{Z}_{N}(\omega)\right|^{p} \mathrm{~d} \omega$ converges w.p. 1 to a finite limit, which implies that, w.p. $1,\left|\hat{Z}_{N}(\cdot)\right|^{p}$ is uniformly integrable.

By (2.1), this shows that $\hat{Z}_{N}$ converges to $Z$ w.p. 1 in the norm topology of the space $L_{p}(\Omega, \mathcal{F}, \mathbb{P})$. It remains to recall that axioms (A1) and (A2) imply that the risk measure $\rho: \mathcal{Z} \rightarrow \mathbb{R}$ is continuous in the norm topology of $\mathcal{Z}=L_{p}(\Omega, \mathcal{F}, \mathbb{P})$ (cf. [7]), and, hence, $\rho\left(\hat{Z}_{N}\right)$ converges to $\rho(Z)$ w.p. 1 .

Convergence w.p. 1 of empirical estimates of law invariant coherent risk measures was studied in [9] by employing techniques based on the Kusuoka representation of law invariant coherent risk measures. The above proof is more direct and does not involve the growth conditions used in [9, Theorem 3.4].

\section{Convergence of statistical estimates of risk averse stochastic programs}

We proceed now to the investigation of the uniform-type convergence of empirical estimates of risk measures. Recall that a sequence of functions $\phi_{k}: \mathbb{R}^{n} \rightarrow \overline{\mathbb{R}}$ is said to epiconverge to a function $\phi$, denoted by $\phi_{k} \stackrel{\mathrm{e}}{\rightarrow} \phi$, if, for any point $\bar{x} \in \mathbb{R}^{n}$, the following two conditions are satisfied (see, e.g. [6, p. 241]):

(i) for any sequence $x_{k}$ converging to $\bar{x}$, it holds that

$$
\liminf _{k \rightarrow \infty} \phi_{k}\left(x_{k}\right) \geq \phi(\bar{x})
$$


(ii) there exists a sequence $x_{k}$ converging to $\bar{x}$ such that

$$
\limsup _{k \rightarrow \infty} \phi_{k}\left(x_{k}\right) \leq \phi(\bar{x})
$$

Consider now the objective function $g(x)=\rho\left(G_{x}\right)$ of the 'true' problem (1.1) and its sample estimate $\hat{g}_{N}(x)=\hat{g}_{N}(x, \omega)$ based on the i.i.d. sample $\xi_{i}=\xi_{i}(\omega), i=1, \ldots, N$. With some abuse of notation, we write $G(x, \omega)$ for the function $G(x, \xi(\omega))$. We now investigate the epiconvergence w.p. 1 of $\hat{g}_{N}$ to $g$. Consider the following conditions.

(C1) For every $x \in \mathbb{R}^{n}$, the random variable $G_{x}(\omega)=G(x, \omega)$ belongs to the space $\mathbb{Z}=$ $L_{p}(\Omega, \mathcal{F}, \mathbb{P}), p \in[1, \infty)$.

(C2) The function $G(x, \omega)$ is random lower semicontinuous, i.e. the epigraphical multifunction $\omega \mapsto$ epi $G(\cdot, \omega)$ is closed valued and measurable.

(C3) For every $\bar{x} \in \mathbb{R}^{n}$, there is a neighborhood $\mathcal{V}_{\bar{x}}$ of $\bar{x}$ and a function $h \in \mathcal{Z}$ such that $G(x, \cdot) \geq h(\cdot)$ for all $x \in \mathcal{V}_{\bar{x}}$.

Some remarks about the above regularity conditions are now in order. Condition (C1) means that, for every $x \in \mathbb{R}^{n}$, the corresponding random variable $G_{x}$ has a finite $p$ th-order moment. For a thorough discussion of random lower-semicontinuous functions and related measurability questions, we refer the reader to [6, Chapter 14]. (Random lower-semicontinuous functions are called normal integrands in [6].) In particular, a set-valued mapping $A: \Omega \rightrightarrows \mathbb{R}^{m}$ is measurable if, for every open set $O \subset \mathbb{R}^{m}$, the set $A^{-1}(O)$ is $\mathcal{F}$-measurable [6, Definition 14.1]. It could be noted that closeness of the epigraph epi $G(\cdot, \omega)$ means that the function $G(\cdot, \omega)$ is lower semicontinuous. A sufficient condition for $G(x, \omega)$ to be random lower semicontinuous is that $G(x, \omega)$ is measurable in $\omega$ for every $x$ and continuous in $x$ for a.e. $\omega$. Such functions are called Carathéodory integrands in [6, Example 14.29].

Theorem 3.1. Let $\rho: \mathcal{Z} \rightarrow \mathbb{R}$ be a law invariant convex risk measure, and let $g(x):=\rho\left(G_{x}\right)$. Suppose that conditions $(C 1)-(C 3)$ hold. Then the function $g(\cdot)$ is lower semicontinuous and $\hat{g}_{N} \stackrel{\mathrm{e}}{\rightarrow} g$ w.p. 1 .

\subsection{Proof of Theorem 3.1}

Note that, since the risk measure $\rho(\cdot)$ is real valued, it follows from $(\mathrm{C} 1)$ that the function $g(\cdot)$ is real valued.

Since the risk measure $\rho$ is convex, it has the dual representation (1.4). Consider a point $\bar{x} \in \mathbb{R}^{n}$. By condition (C3) we have, for any $\zeta \in \mathfrak{A}$ and $x \in \mathcal{V}_{\bar{x}}, \zeta(\cdot) G(x, \cdot) \geq \zeta(\cdot) h(\cdot)$. Moreover, since $h \in \mathbb{Z}$ and $\zeta \in \mathbb{Z}^{*}$, then $\int_{\Omega} \zeta(w) h(\omega) \mathrm{dP}(\omega)$ is finite. Hence, by Fatou's lemma, it follows that, for a sequence $x_{k} \rightarrow \bar{x}$,

$\liminf _{k \rightarrow \infty} \int_{\Omega} \zeta(\omega) G\left(x_{k}, \omega\right) \mathrm{d} \mathbb{P}(\omega) \geq \int_{\Omega} \liminf _{k \rightarrow \infty} \zeta(\omega) G\left(x_{k}, \omega\right) \mathrm{d} \mathbb{P}(\omega) \geq \int_{\Omega} \zeta(\omega) G(\bar{x}, \omega) \mathrm{d} \mathbb{P}(\omega)$,

where the last inequality follows by lower semicontinuity of $G(\cdot, \omega)$ (condition $(\mathrm{C} 2)$ ). That is, the function $x \mapsto \int_{\Omega} \zeta(\omega) G(x, \omega) \mathrm{d} \mathbb{P}(\omega)-\rho^{*}(\zeta)$ is lower semicontinuous. Since, by (1.4), $g(x)=\rho\left(G_{x}\right)$ is given by a maximum of such functions, it follows that $g(x)$ is also lower semicontinuous.

In order to show that $\hat{g}_{N} \stackrel{\text { e }}{\rightarrow} g$ w.p. 1 , we need to verify conditions (i) and (ii). That is, to verify condition (i), we have to show that there exists a set $\Delta \subset \Omega$ of measure 0 such that, for 
any point $\bar{x} \in \mathbb{R}^{n}$ and any sequence $x_{N}$ converging to $\bar{x}$, it holds that

$$
\liminf _{N \rightarrow \infty} \hat{g}_{N}\left(x_{N}, \omega\right) \geq g(\bar{x}) \quad \text { for all } \omega \in \Omega \backslash \Delta .
$$

Note that the set $\Delta$ should not depend on $\bar{x}$. Similarly for condition (ii).

To verify condition (i), we proceed as follows. For some sequence $\gamma_{k} \downarrow 0$ of positive numbers, consider $V_{k}:=\left\{x \in \mathbb{R}^{n}:\|x-\bar{x}\|<\gamma_{k}\right\}$, and let

$$
g_{k}(\omega):=\inf _{x \in V_{k}} G(x, \omega), \quad k \in \mathbb{N} .
$$

Since $G(x, \omega)$ is random lower semicontinuous (condition $(\mathrm{C} 2)$ ), then $g_{k}(\omega)$ is measurable (cf. [6]). By (C3) we have $g_{k}(\cdot) \geq h(\cdot)$ for all large enough $k$ (such that $V_{k} \subset \mathcal{V}_{\bar{x}}$ ). Of course, we also have $G(x, \cdot) \geq g_{k}(\cdot)$ for any $x \in V_{k}$. It follows that $g_{k} \in \mathcal{Z}$. Consider the dual representation (1.4) of $\rho$, and let

$$
\bar{\zeta} \in \arg \max _{\zeta \in \mathfrak{A}}\left\{\mathbb{E}[\zeta(\omega) G(\bar{x}, \omega)]-\rho^{*}(\zeta)\right\}
$$

(note that such a maximizer exists [7]). That is, $\bar{\zeta} \in \mathfrak{A}$ and

$$
g(\bar{x})=\mathbb{E}[\bar{\zeta}(\omega) G(\bar{x}, \omega)]-\rho^{*}(\bar{\zeta}) .
$$

Since $g_{k} \in \mathbb{Z}$, we have, by (1.4),

$$
\rho\left(g_{k}\right) \geq \mathbb{E}\left[\bar{\zeta}(\omega) g_{k}(\omega)\right]-\rho^{*}(\bar{\zeta}) .
$$

By condition $(\mathrm{C} 3), \bar{\zeta}(\cdot) g_{k}(\cdot)$ is bounded from below, on a neighborhood of $\bar{x}$, by the integrable function $\bar{\zeta}(\cdot) h(\cdot)$, and, hence, applying Fatou's lemma we have

$$
\liminf _{k \rightarrow \infty} \mathbb{E}\left[\bar{\zeta}(\omega) g_{k}(\omega)\right] \geq \mathbb{E}\left[\liminf _{k \rightarrow \infty} \bar{\zeta}(\omega) g_{k}(\omega)\right]
$$

and, by the lower semicontinuity of $G(\cdot, \omega)$,

$$
\mathbb{E}\left[\liminf _{k \rightarrow \infty} \bar{\zeta}(\omega) g_{k}(\omega)\right]-\rho^{*}(\bar{\zeta}) \geq \mathbb{E}[\bar{\zeta}(\omega) G(\bar{x}, \omega)]-\rho^{*}(\bar{\zeta})=g(\bar{x})
$$

We obtain

$$
\liminf _{k \rightarrow \infty} \rho\left(g_{k}\right) \geq g(\bar{x}) .
$$

Now let us choose $\varepsilon>0$. By (3.2), there exists $\bar{k}=\bar{k}(\varepsilon)$ such that

$$
\rho\left(g_{\bar{k}}\right) \geq g(\bar{x})-\varepsilon .
$$

Let $\hat{\rho}_{N}\left(g_{\bar{k}}\right)$ be the empirical estimate of $\rho\left(g_{\bar{k}}\right)$ based on the same sample as the sample used for the estimate $\hat{g}_{N}(\cdot)$, i.e. $\hat{\rho}_{N}\left(g_{\bar{k}}\right)=\rho\left(\hat{H}_{N}\right)$, where $\hat{H}_{N}$ is the empirical CDF of the sample $Y_{i}=\inf _{x \in V_{\bar{k}}} \bar{G}\left(x, \xi_{i}\right), i=1, \ldots, N$. By Theorem 2.1 we have $\hat{\rho}_{N}\left(g_{\bar{k}}\right) \rightarrow \rho\left(g_{\bar{k}}\right)$ w.p. 1 as $N \rightarrow \infty$. Hence, for a.e. $\omega \in \Omega$, there exists $\bar{N}_{\bar{x}}(\omega)$ such that $\hat{\rho}_{N}\left(g_{\bar{k}}\right) \geq \rho\left(g_{\bar{k}}\right)-\varepsilon$ for all $N \geq \bar{N}_{\bar{x}}(\omega)$. Together with (3.3) this implies that

$$
\hat{\rho}_{N}\left(g_{\bar{k}}\right) \geq g(\bar{x})-2 \varepsilon
$$

for a.e. $\omega \in \Omega$ and $N \geq \bar{N}_{\bar{x}}(\omega)$. 
For any $x \in V_{\bar{k}}$, we have (for the same random sample) that the empirical CDF of $G(x, \cdot)$ dominates the empirical CDF of $g_{\bar{k}}(\cdot)$, and, hence (cf. [8, Theorem 6.28]),

$$
\hat{g}_{N}(x)=\hat{\rho}_{N}\left(G_{x}\right) \geq \hat{\rho}_{N}\left(g_{\bar{k}}\right) \text {. }
$$

By (3.4), it follows that

$$
\inf _{x \in V_{\bar{k}}} \hat{g}_{N}(x) \geq g(\bar{x})-2 \varepsilon
$$

for a.e. $\omega \in \Omega$ and $N \geq \bar{N}_{\bar{x}}(\omega)$. That is, there exist $\bar{N}(\omega)$, a set $\Upsilon \subset \Omega$ of measure 0 , and a neighborhood $V$ (both depending on $\bar{x}$ and $\varepsilon$ ) such that

$$
\hat{g}_{N}(x) \geq g(\bar{x})-2 \varepsilon
$$

for all $N \geq \bar{N}(\omega), x \in V$, and $\omega \in \Omega \backslash \Upsilon$. It follows that there exists a countable number of points $x_{1}, \ldots$ in $\mathbb{R}^{n}$, with corresponding neighborhoods $V_{1}, \ldots$ covering $\mathbb{R}^{n}$. Let $\Upsilon_{1}, \ldots$ be the corresponding sets of measure 0 , and let $\bar{\Upsilon}:=\bigcup_{i=1}^{\infty} \Upsilon_{i}$. Note that the set $\bar{\Upsilon}$ has measure 0 , and that $\bar{\Upsilon}$ depends on $\varepsilon$, but not on a particular point of $\mathbb{R}^{n}$. It follows that, for any $\bar{x} \in \mathbb{R}^{n}$, there is a neighborhood $W$ and $N^{*}(\omega)$ such that (3.5) holds for all $x \in W, N \geq N^{*}(\omega)$, and $\omega \in \Omega \backslash \bar{\Upsilon}$.

Consequently, for any point $\bar{x}$ and a sequence $x_{N}$ converging to $\bar{x}$, we have

$$
\liminf _{N \rightarrow \infty} \hat{g}_{N}\left(x_{N}, \omega\right) \geq g(\bar{x})-2 \varepsilon
$$

for all $\omega \in \Omega \backslash \bar{\Upsilon}$. Now choose a sequence of positive numbers $\varepsilon_{i} \downarrow 0$ and let $\bar{\Upsilon}_{i}$ be the corresponding sets of measure 0 . Set $\Delta:=\bigcup_{i=1}^{\infty} \bar{\Upsilon}_{i}$. Then (3.6) implies that (3.1) holds for any $\bar{x} \in \mathbb{R}^{n}$ and any sequence $x_{N}$ converging to $\bar{x}$. This proves that condition (i) holds for a.e. $\omega \in \Omega$.

In order to verify condition (ii), we need the following result.

Lemma 3.1. There exists a countable set $\mathscr{D} \subset \mathbb{R}^{n}$ such that, for any point $\bar{x} \in \mathbb{R}^{n}$, there exists a sequence $x_{k} \in \mathscr{D}$ converging to $\bar{x}$ and

$$
\limsup _{k \rightarrow \infty} g\left(x_{k}\right) \leq g(\bar{x}) .
$$

Proof. Such a set $D$ can be constructed as follows. Let $A \subset \mathbb{R}$ be a countable and dense subset of $\mathbb{R}$. Consider level sets $\mathcal{L}_{a}:=\left\{x \in \mathbb{R}^{n}: g(x) \leq a\right\}$, and let $D_{a}, a \in A$, be a countable and dense subset of $\mathcal{L}_{a}$. (Of course, some of the sets $\mathcal{L}_{a}$ and $D_{a}$ can be empty.) Define $\mathscr{D}:=\bigcup_{a \in A} D_{a}$. Clearly, the set $\mathscr{D}$ is countable. Condition (3.7) also holds. Indeed, let $a_{k} \in A$ be a monotonically decreasing sequence converging to $g(\bar{x})$. Note that $\bar{x} \in \mathcal{L}_{a_{k}}$ for all $k$. Therefore, there exists a point $x_{k} \in D_{a_{k}}$ such that $\left\|x_{k}-\bar{x}\right\| \leq 1 / k$. We have then $x_{k} \rightarrow \bar{x}$ and $g\left(x_{k}\right) \leq a_{k}$, and, hence, (3.7) follows, completing the proof of the lemma.

Let $\mathscr{D}$ be a set specified in Lemma 3.1. Consider a point $\bar{x} \in \mathbb{R}^{n}$, and let $x_{k} \in \mathscr{D}$ be a sequence of points converging to $\bar{x}$ such that (3.7) holds. For a given $x \in \mathbb{R}^{n}$, it follows by Theorem 2.1 that $\hat{g}_{N}(x)$ converges to $g(x)$ w.p. 1. That is, there exists a set $\Upsilon_{x}$ of measure 0 such that $\hat{g}_{N}(x, \omega)$ converges to $g(x)$ for every $\omega \in \Omega \backslash \Upsilon_{x}$. Consider the set $\tilde{\Upsilon}:=\bigcup_{x \in D} \Upsilon_{x}$. Since the set $\mathscr{D}$ is countable, the set $\tilde{\Upsilon}$ has measure 0 . The function $\hat{g}_{N}(x, \omega)$ converges to $g(x)$ for every $x \in \mathscr{D}$ and $\omega \in \Omega \backslash \tilde{\Upsilon}$. Hence, there is a sequence $N_{k}=N_{k}(\omega)$ of positive integers such that, for all $k$,

$$
\left|\hat{g}_{N_{k}}\left(x_{k}, \omega\right)-g\left(x_{k}\right)\right|<\frac{1}{k} \quad \text { for all } \omega \in \Omega \backslash \tilde{\Upsilon} .
$$


Now let $x_{N}^{\prime}$ be a sequence of points such that $x_{N_{k}}^{\prime}=x_{k}$ for all $k$. We then have $x_{N_{k}}^{\prime} \rightarrow \bar{x}$ and, by (3.7) and (3.8),

$$
\limsup _{k \rightarrow \infty} \hat{g}_{N_{k}}\left(x_{N_{k}}^{\prime}, \omega\right) \leq g(\bar{x}) \quad \text { for all } \omega \in \Omega \backslash \tilde{\Upsilon}
$$

This shows that condition (ii) holds w.p. 1, completing the proof of Theorem 3.1.

For the expectation operator, i.e. when $\rho(\cdot)=\mathbb{E}(\cdot)$, the epiconvergence result of Theorem 3.1 was proved in [1].

Suppose now that, for a.e. $\omega$, the function $G(\cdot, \omega)$ is convex and let $\rho: \mathcal{Z} \rightarrow \mathbb{R}$ be a law invariant convex risk measure. Then the functions $g(x)=\rho\left(G_{x}\right)$ and $\hat{g}_{N}(x)$ are also convex. It is known that if $\phi_{k}: \mathbb{R}^{n} \rightarrow \overline{\mathbb{R}}$ is a sequence of convex functions and $\phi: \mathbb{R}^{n} \rightarrow \overline{\mathbb{R}}$ is a convex lower-semicontinuous function such that its domain has a nonempty interior, then the following conditions are equivalent: (a) $\phi_{k} \stackrel{\mathrm{e}}{\rightarrow} \phi$, (b) there exists a dense subset $\mathcal{D}$ of $\mathbb{R}^{n}$ such that $\phi_{k}(x) \rightarrow \phi(x)$ for all $x \in \mathcal{D}$, (c) $\phi_{k}(\cdot)$ converges uniformly to $\phi(\cdot)$ on every compact set $C \subset \mathbb{R}^{n}$ that does not contain boundary points of dom( $\phi$ ) (cf. [6, Theorem 7.17]). These together with Theorem 2.1 imply the following result in a straightforward way. Recall that, by condition $(\mathrm{C} 1)$, the function $g(x)$ is real valued and, hence, $\operatorname{dom}(g)=\mathbb{R}^{n}$.

Theorem 3.2. Let $\rho: \mathcal{Z} \rightarrow \mathbb{R}$ be a law invariant convex risk measure, and let $g(x):=\rho\left(G_{x}\right)$. Suppose that condition $(C 1)$ holds, and, for a.e. $\omega$, the function $G(\cdot, \omega)$ is convex. Then the functions $g(\cdot)$ and $\hat{g}_{N}(\cdot)$ are convex and $\hat{g}_{N}(\cdot)$ converges w.p. 1 uniformly to $g(\cdot)$ on every compact set.

The results of Theorem 3.1 can be applied in a straightforward way to investigate the consistency of the sample approximation (1.3) of the 'true' problem (1.1).

Theorem 3.3. Let $\rho: \mathcal{Z} \rightarrow \mathbb{R}$ be a law invariant convex risk measure. Suppose that conditions (C1)-(C3) hold and that the set $\mathcal{X}$ is nonempty and compact. Then the optimal value of the SAA problem (1.3) converges w.p. 1 to the optimal value of the 'true' problem (1.1), and the distance from an optimal solution of (1.3) to the set of optimal solutions of (1.1) converges w.p. 1 to 0 as $N \rightarrow \infty$

Proof. Since the function $g(x)$ is lower semicontinuous and the set $\mathcal{X}$ is compact, problem (1.1) has a nonempty set $\mathfrak{S}$ of optimal solutions and its optimal value $\vartheta^{*}$ is finite. Since $\hat{g}_{N} \stackrel{\text { e }}{\rightarrow} g$ w.p. 1, it follows that the optimal value $\hat{\vartheta}_{N}$ of problem (1.3) converges w.p. 1 to $\vartheta^{*}$, and the distance from an optimal solution of (1.3) to the set $\mathfrak{S}$ converges w.p. 1 to 0 as $N \rightarrow \infty$ (cf. [6, Chapter 7]).

The assumption of compactness of the set $\mathcal{X}$ in Theorem 3.3 is needed in order to ensure existence of optimal solutions of the true and SAA optimization problems, and that optimal solutions of the SAA problems stay w.p. 1 in a bounded set. In some problems this can be verified by ad-hoc methods.

For risk neutral stochastic programming problems, the epiconvergence approach was used in [4] to study asymptotic consistency of the corresponding statistical estimators.

\section{Acknowledgements}

This research was partly supported by the NSF award DMS-0914785 and ONR award N000140811104. 


\section{References}

[1] Artstein, Z. and Wets, R. J. B. (1996). Consistency of minimizers and the SLLN for stochastic programs. J. Convex Anal. 2, 1-17.

[2] Artzner, P., Delbaen, F., Eber, J.-M. and Heath, D. (1999). Coherent measures of risk. Math. Finance 9, 203-228.

[3] Billingsley, P. (1995). Probability and Measure, 3rd edn. John Wiley, New York.

[4] Dupačová, J. AND Wets, R. J. B. (1988). Asymptotic behavior of statistical estimators and of optimal solutions of stochastic optimization problems. Ann. Statist. 16, 1517-1549.

[5] Föllmer, H. And Schied, A. (2004). Stochastic Finance: An Introduction in Discrete Time, 2nd edn. Walter de Gruyter, Berlin.

[6] Rockafellar, R. T. and Wets, R. J. B. (1998). Variational Analysis. Springer, Berlin.

[7] Ruszczyński, A. AND Shapiro, A. (2006). Optimization of convex risk functions. Math. Operat. Res. 31, 433-452.

[8] Shapiro, A., Dentcheva, D. And Ruszczyński, A. (2009). Lectures on Stochastic Programming: Modeling and Theory. SIAM, Philadelphia.

[9] Wozabal, D. and Wozabal, N. (2009). Asymptotic consistency of risk functionals. J. Nonparametric Statist. 21, 977-990. 